\title{
Valores y normas en Hans Kelsen y Max Ernst Mayer
}

\author{
Miguel Herszenbaun \\ CONICET/UBA/CIF/GEK
}

herszen@hotmail.com

Recibido 12/06/2018

Aprobado 27/07/2018

\section{Resumen}

En el presente trabajo contrapongo el modelo kelseniano según el cual se concibe al derecho positivo como un sistema de valores relativos al modelo propuesto por Max Ernst Mayer, de acuerdo con el cual la norma jurídica debe sostenerse en y concordar con normas culturales pre-jurídicas. Sostengo que la propuesta de Mayer puede ser entendida como una herramienta superadora del esquema dicotómico planteado por Hans Kelsen (dicotomía entre valores relativos/valores absolutos), pero que a su vez se demuestra insuficiente para dar respuesta a las propias preguntas que Max Ernst Mayer pretende enfrentar. En este sentido, me propongo demostrar que es necesario integrar un rasgo dialéctico a la dinámica por medio de la cual la comunidad genera pluralidad de fuentes normativas y a la dinámica por medio de la cual las diferentes normas interactúan, so pena de no ser capaz de explicar la validez de las normas jurídicas y culturales ni la dinámica por medio de las cuales unas derogan a las otras.

Palabras clave: norma - jurídica - cultural - valor

\section{Abstract}

In the following paper, I compare Kelsen's model, according to which positive Law is understood as a system of relative values, with Max Ernst Mayer's model, in which legal norms must be based on and agree with pre-legal culture norms. I claim that Mayer's proposition can be understood as a tool that goes beyond Kelsen's dichotomic scheme, but at the same time, it proves to be insufficient for answering main questions raised by Mayer. Accordingly, I intent to prove that it is necessary to 
integrate a dialectic character to the dynamic by which the community produces multiple normative sources and to the dynamic by which different norms interact, on pain of not being able to explain the validity of legal and culture norms and the dynamics by which norms derogate each other.

Keywords: Norm - Legal - Culture - Value

\section{I- Introducción}

Durante las últimas décadas del siglo XIX y las primeras del siglo XX el concepto de valor fue un elemento clave de la interpretación filosófica de las ciencias humanas y una pieza clave del desarrollo epistemológico, fundamentalmente para la tradición neokantiana. En congruencia, entre la bibliografía especializada se ha destacado el lugar que en el pensamiento de Windelband, Rickert, Cassirer, entre otros, se ha dado al concepto de valor, al punto tal de que se ha admitido llamar también a dichas filosofías de la cultura teorías del valor ${ }^{1}$. A pesar de que la posible raigambre kantiana/neokantiana de la obra de Kelsen ha sido debidamente advertida ${ }^{2}$ y aunque se ha atendido al concepto de valor en Kelsen ${ }^{3}$, amerita ser señalado que la bibliografía

\footnotetext{
${ }^{1}$ En congruencia con esto, según Sprenger, el neokantismo de la escuela de Baden comienza a concebir a la filosofía como una teoría de la cultura que tiene por tarea superar a la vieja metafísica. En esta teoría de la cultura, el concepto de valor termina por tener un lugar fundamental. Cfr. Sprenger, Gerhard, "Die Wertlehre des Badener Neukantianismus und ihre Ausstrahlungen in die Rechtsphilosophie“, Alexy, R., Meyer, L., Paulson, S., Sprenger, G., Neukantianismus und Rechtsphilosophie, Nomos Verlagsgesselschaft, Baden-Baden, 2002, pp. 159 y ss. Según Luft, la nota distintiva del neokantismo no es sólo la apropiación por parte de Cohen del método kantiano (método que después sería reelaborado por Natorp), sino también la propuesta original de Cohen de convertir a la crítica de la razón kantiana en una crítica de la cultura. Según Luft, este elemento central del neokantismo perdura hasta incluso en la obra de Cassirer. Cfr. Sebastian Luft, The Space of Culture: Towards a Neo-Kantian. Philosophy of Culture (Cohen, Natorp, and Cassirer), Oxford, Oxford Scholarship Online, 2015. 18-19, 20-21. Esto permite conformar una constelación que vincula a las filosofías de la cultura, las teorías del valor y la tradición neokantiana.

${ }^{2}$ Véase por ejemplo, Paulson, Stanley, "The Neo-Kantian Dimension of Kelsen's Pure Theory of Law", Oxford Journal of Legal Studies, Vol 12, No 3, 1992, pp. 311-332. Paulson, Stanley, "La alternativa kantiana de Kelsen: Una crítica", DOXA, n. 9, 1991, pp. 173-187. Hammer, Stefan, "A Neo-Kantian Theory of Legal Knowledge in Kelsen's Pure Theory of Law?", Paulson, Stanley, Litschewski Paulson, Bonnie, Normativity and Norms. Critical Perspectives on Kelsenian Themes, Oxford, Clarendon Press, 1998, pp. 177-194. Schmill, Ulises, "Algunas influencias de Hermann Cohen en Hans Kelsen", Isonomía, no. 21, México, oct. 2004, pp. 117-155.

${ }^{3}$ Stanley Paulson se cuenta entre quienes han insertado a Kelsen en la tradición neokantiana, dándole una especial importancia en esta inserción al concepto de valor. Paulson sostiene que Kelsen, siguiendo a Rickert, establece un dualismo entre el mundo de la facticidad y el mundo del valor. Según el autor, Kelsen intenta establecer un puente entre ambos a través de los conceptos de sentido subjetivo y sentido objetivo del acto creador de derecho. Cfr. Paulson, Stanley, "Faktum/Wert-Distinktion, Zwei-
} 
especializada no ha prestado especial interés a una posible clave de lectura de su filosofía del Derecho como una teoría del valor. Por supuesto, la teoría del derecho de Kelsen, leída como teoría del valor, supondría centrarnos fundamentalmente en el derecho como fuente de valores, desplazando otras posibles fuentes suyas y supondría, por otra parte, considerar otras teorías competidoras que también conciben al derecho como fuente de valores, como la teoría de Max Ernst Mayer.

En el presente trabajo me propongo contraponer dos modelos a través de los cuales se piensa al derecho como un sistema de valores. El primer modelo a considerar es la concepción kelseniana del derecho. De acuerdo con este modelo, el derecho positivo se presenta como un sistema de valores convencionales, en oposición a un hipotético sistema de valores absolutos (de origen natural). El segundo modelo es el propuesto por Max Ernst Mayer, en el que se establece una dicotomía entre norma cultural y norma jurídica. Cabe señalar que la novedad de la presente propuesta radica en que la Teoría pura del derecho de Kelsen no ha sido estudiada como una teoría del valor y en que tampoco se encuentra en la bibliografía especializada un trabajo comparativo entre la obra de Kelsen y Max Ernst Mayer. Me propongo demostrar que el esquema de Mayer puede ser interpretado como una manera de evadir el esquema dicotómico planteado por Kelsen. La superación de esta dicotomía consistiría en concebir al derecho como un sistema de valores convencionales relativos, pero que puedan ser evaluados axiológicamente por pautas extra-jurídicas, a través de enunciados que tengan valor comunitario (incluso jurídico). Ahora bien, como respuesta a Mayer, en la última sección del presente trabajo me propongo demostrar que su esquema requiere introducir una caracterización dialéctica de la dinámica de interacción entre normas de diferente fuente. Sin esta caracterización dialéctica, Mayer es incapaz de explicar las diferentes fuentes normativas, la validez de sus normas, ni la aptitud de las normas jurídicas para modificar normas culturales de la comunidad. 


\section{II- Valores relativos y valores absolutos en la Teoría pura del derecho de Kelsen}

La Teoría pura del derechode Kelsen es, muy probablemente, la obra de filosofía jurídica más influyente del siglo $X X^{4}$. En dicha obra Kelsen se propone trazar los lineamientos fundamentales para llevar a cabo una ciencia del derecho en sentido estricto. Como se sabe, la pretensión positivista de Kelsen -que lo pone en diálogo con el positivismo del siglo XIX y el positivismo lógico del Círculo de Viena- exige que toda ciencia tenga un objeto de investigación cuyo conocimiento pueda ser verificado empíricamente. Sólo este requisito puede separar la ciencia de los desatinos de la metafísica;pero exige la delimitación precisa y nítida de dicho objeto de investigación ${ }^{5}$.

Tal tarea de delimitación se lleva a cabo desde distintos niveles y abriendo diferentes frentes de análisis y disputa. Kelsen tendrá que definir a la norma jurídica incluyendo en ella un aspecto fáctico, empíricamente verificable que, a mi criterio, será el elemento central para definir a su teoría como positivista: la norma puesta ${ }^{6}$. Esta tarea de delimitación pondrá a la teoría pura kelseniana en franca discusión con el iusnaturalismo ${ }^{7}$. Sin embargo, el elemento fáctico no es el elemento definitorio de lo normativo: la noción de validez será característica de lo normativo, cuestión que abrirá el camino a un nuevo terreno de preguntas que la Teoría pura debe enfrentar. ${ }^{8}$

Al proponerse delimitar el objeto de investigación de una ciencia jurídica, Kelsen debe separar el terreno de lo jurídico de otros terrenos inmiscuidos en el quehacer legislativo y jurisprudencial, como por ejemplo el ámbito de la moral, la religión, la política, la historia, etc. Uno de los elementos de mayor importancia en esta tarea de delimitación es identificar que la ciencia jurídica debe versar sobre normas. El segundo paso es distinguir normas puestas o positivas de normas supuestas. La nota distintiva de la norma puesta es que ha sido creada por un acto creador de derecho en

\footnotetext{
${ }^{4}$ Como es sabido, Hans Kelsen publicó dos ediciones de la Teoría pura del derecho (1934 y 1960) que no sólo difieren en extensión, sino también en contenido. El presente trabajo no se propone considerar las diferencias entre ambas obras. Las referencias contenidas en este trabajo remiten a la traducción de la Teoría pura del derecho publicada por Eudeba, que consiste en una traducción al español de la traducción al francés del original de 1934 (versión ampliada, también publicada en el año 1960). Kelsen, Hans, Teoría pura del derecho, Eudeba, Buenos Aires, 2015.

5 Ibid., p. 19

6 Ibid., p. 21

${ }^{7}$ Ibid., pp. 23, 40-41

${ }^{8}$ La noción de validez, aunque central a la hora de definir el terreno de lo normativo o prescriptivo, exigirá, a criterio de Kelsen, postular la problemática idea de una norma fundamental y abordar la dificultosa relación entre validez y eficacia. Sobre esto, volveremos más adelante.
} 
un tiempo y espacio determinados. ${ }^{9}$ En otras palabras, la norma positiva debe haber sido creada a través de un acto verificable, contrastable, dándole esto -a mi criterioel carácter positivista a la teoría: habrá ciencia del derecho, cuando nos ocupemos de estudiar normas (describir enunciados normativos por medio de reglas del derecho) creadas por actos llevados a cabo en algún tiempo y espacio -lo que hace al acto verificable empíricamente- y no cuando nos ocupemos de investigar presuntas normas de existencia dudosa.

Este primer elemento pone a la teoría pura de Kelsen en franca contraposición con el iusnaturalismo. Como sabemos, la escuela iusnaturalista sostiene que junto al derecho positivo creado por la voluntad y convención humana, existe un orden normativo de origen natural -esto es, no creado por la voluntad humana y, por tanto, inderogable, perpetuo y universalmente válido- de carácter perfecto ${ }^{10}$. Su perfección y suma justicia pondría al derecho natural en preeminencia frente al derecho positivo, lo que implicaría que éste debe tomar a aquél como parámetro. Es decir, el derecho natural sería el parámetro a partir del cual juzgamos al derecho positivo y, en consecuencia, su fuente de validez.

La manera en que Kelsen ha separado la norma puesta o positiva de la norma supuesta a través del acto creador ejecutado en el tiempo y en el espaciosupone trazar una dicotomía entre una teoría positivista del derecho y una concepción iusnaturalista. Si la norma objeto de investigación de la ciencia jurídica debe haber sido creada por un ser humano en un tiempo y espacio determinados, el sistema normativo postulado y defendido por el iusnaturalismo queda fuera de toda posible consideración científica. El iusnaturalismo podrá postular la existencia de un sistema normativo perfectamente justo, pero de ninguna forma se trata de una norma creada a través de un acto verificable empíricamente. La consideración de dicho sistema normativo cuyo acto de creación está fuera del tiempo y el espacio $-y$ en consecuencia, más allá de toda verificación posible- debe quedar, por tanto, en el terreno de los presuntos conocimientos metafísicos y no en el terreno de la ciencia.

\footnotetext{
${ }^{9}$ Ibid., p. 31

${ }^{10}$ Strauss, Leo, "Sobre la ley natural", Persecución y el arte de escribir y otros ensayos de filosofía política, Edicions Alfons El Magnánim, Valencia, 1996, pp. 134-135
} 
La definición kelseniana de la norma no estaría completa sin incluiruna consideración sobre la validez y la eficacia. Si bien nuestro objetivo no es desarrollar la noción de norma en Kelsen, sino considerar el sistema de valores que se presenta en un sistema normativo, es necesario mencionar la noción de validez.

Kelsen define a la validez como el modo de existencia de la norma. Podríamos decir, así como las sustancias extensas (objetos espaciotemporales) tienen como modo de existencia ocupar espacio y tiempo (es decir, existir para un cuerpo es ocupar espacio y durar en el tiempo), el modo de la existencia de una norma, de una prescripción, es valer, regir ${ }^{11}$. Una norma es norma y existe como tal en tanto vale lo que ella prescribe, en tanto ordena legítimamente. Como puede apreciarse, la validez es un elemento central de la norma. Es, en otras palabras, lo que convierte al acto creador de derecho en, precisamente, un acto creador de derecho, es decir, de norma. Lo que hace que el acto llevado a cabo por el legislador sea un acto creador de derecho es su relación con normas precedentes. Otras normas definen cómo se ha de crear el derecho y el acto en cuestión será creador de derecho en tanto sea ejecutado en concordancia con dichas normas. Al depender la validez de una determinada norma de normas precedentes que establecen los procedimientos para crear válidamente derecho, se conforma una estructura normativa sistemática y jerárquica en la cual la validez se deduce de manera descendente de normas de jerarquía superior. La norma, en consecuencia, tendrá validez y existirá como norma en tanto reciba su validez de normas superiores, es decir, en tanto haya sido creada en concordancia con normas de nivel superior en dicha estructura jerárquica. Los elementos normativos valdrán como tales elementos normativos, como partes del sistema normativo, en tanto y en cuanto reciban su validez de los elementos normativos superiores y todos ellos formarán parte de un mismo sistema normativo en tanto puedan rastrear su validez a una fuente común y central.

Dicha fuente central de validez es la primera constitución del sistema normativo, cuya validez depende a su vez de una norma supuesta que Kelsen llama norma fundamental ${ }^{12}$. La norma fundamental es, en oposición a la norma puesta, una mera norma supuesta. Es decir, no se trata de un elemento normativo positivo, de una

\footnotetext{
${ }^{11}$ Kelsen, Hans, Teoría pura del derecho, ob cit.,, p. 31.

12 Ibid., p. 34-35.
} 
norma positiva, creada por un ser humano en un tiempo y espacio determinados. Se trata de una suposición o hipótesis postulada por el científico ${ }^{13}$. Se supone una norma que le atribuiría validez a la constitución, es decir, a la norma positiva de mayor jerarquía en el sistema normativo. El estatuto exacto de la norma fundamental al interior de la filosofía de Kelsen es complejo ${ }^{14}$. Para nuestros fines, basta con indicar

13 "La voluntad del primer constituyente debe ser considerada, pues, como poseedora de un carácter normativo, y de esta hipótesis fundamental debe partir toda investigación científica sobre el orden jurídico considerado". "Sólo la hipótesis de una norma fundamental permite conferir un sentido jurídico a los materiales empíricos que se presentan al examen del jurista y considerarlos como formando un sistema de normas". "Es la hipótesis que permite a la ciencia jurídica considerar al derecho como un sistema de normas válidas. Todas las proposiciones por las cuales esta ciencia describe su objeto están fundadas sobre el supuesto de que la norma fundamental es una norma válida". Ibid., , p. 113. De manera similar, "para atribuir a ciertos hechos la calidad de hechos creadores de normas válidas es necesario suponer la existencia de una norma fundamental." ibid., pp. 34-35.

${ }^{14}$ La caracterización precisa de la norma fundamental al interior de la filosofía de Kelsen es un tema que por sí mismo exigiría una investigación autónoma. Creo que la dificultad de precisar esta cuestión no depende únicamente de los cambios de perspectiva que el propio Kelsen habría adoptado (para considerar estos cambios, véase Marí, Enrique, "Racionalidad e imaginario social en el discurso del orden, Doxa, n 3, 1986, pp. 107-110.), sino que se deben también -en mi opinión- a la falta de explicitación de supuestos filosóficos implícitos que parecerían estar operando en el pensamiento de Kelsen (y que resultan, a su vez, especialmente difíciles de precisar). Por ejemplo, como ya hemos indicado en citas precedentes, en la primera edición de la Teoría pura del derecho, Kelsen sugiere que la norma fundamental es una hipótesis. Todo indicaría que en este contexto debe entenderse que se trata de una hipótesis postulada por el científico que investiga al sistema normativo. De esta manera, ante un sistema normativo eficaz, el científico se encuentra autorizado a postular, a suponer, la existencia de una norma fundamental que indica que el sistema es válido (que le da el sentido de ser norma a los elementos que él estudia). Aquí no nos interesa desarrollar los problemas que esta primera caracterización de la norma fundamental podría presentar. Nos basta con contraponerla a la caracterización que encontramos de ella en la segunda edición de la Teoría pura del derecho.

Allí Kelsen introduce un término que es de especial importancia, pues pone al concepto de norma fundamental en relación con una determinada tradición filosófica: Kelsen la llama presupuesto lógicotrascendental (Kelsen, Hans, Teoría pura del derecho, trad. R. Vernengo, UNAM, México, 1982, pp. 208, "transzendental-logische Voraussetzung"). Este particular adjetivo hace suponer al lector avezado que Kelsen arrima su noción de norma fundamental a la tradición kantiana o neokantiana. Si a esto se suman las múltiples referencias a Kant que encontramos en ambas ediciones de la Teoría pura, las sospechas se hacen más sólidas.

Kant utiliza explícitamente el concepto de hipótesis trascendental ("transscendentale Hypothese" sic) en el contexto de la "doctrina del método" de la Crítica de la razón pura (A772-3/B800-1 y A779/B807). En este contexto, Kant explica que la hipótesis trascendental, como una explicación hiperfísica de la realidad, no tiene uso legítimo desde el punto de vista del conocimiento teórico ni explica, en sentido estricto, nada. Pues lo desconocido -lo que se encuentra más allá de la experiencia- nada puede aportar a la explicación y comprensión de la experiencia. La hipótesis trascendental no tiene uso dogmático, pero sí problemático: se la puede utilizar como instrumento en una disputa, en defensa de los intereses prácticos de la razón. Véase Eisler, Lexicon. Online: http://www.textlog.de/32403.html

Parece difícil suponer que Kelsen esté caracterizando a la norma fundamental como una idea trascendental de la razón. Con lo cual, no puede tomarse a la norma fundamental como hipótesis trascendental en el sentido estrictamente kantiano. Salvo que entendamos que para Kelsen la norma fundamental juegue un papel equivalente en el terreno del conocimiento del derecho al que las ideas trascendentales juegan en la filosofía kantiana en el terreno de los intereses prácticos de la razón. En otras palabras, así como las ideas trascendentales no brindan conocimiento teórico pero sí posibilitan el terreno del conocimiento práctico (en concordancia con los intereses prácticos de la razón), la norma 
que Kelsen señala que el científico estaría autorizado a postular la existencia de tal norma fundamental cuando el sistema normativo muestra cierto grado de eficacia (i.e. de acatamiento o aplicación) ${ }^{15}$.

La oposición que Kelsen plantea entre derecho positivo y derecho natural corre en paralelo a la oposición entre norma puesta y norma supuesta, como así también corre en paralelo a la contraposición entre un sistema de valores absolutos y un sistema de valores relativos. En su crítica al iusnaturalismo, Kelsen vincula la noción de norma a los conceptos de voluntad y valor. Una norma impone una prescripción, lo que debe suponer una ponderación o valoración sobre ciertas conductas humanas y una voluntad legisladora ${ }^{16}$. Estos elementos permiten formular una crítica al iusnaturalismo, pues la postulación de un derecho natural equivaldría a postular finalidades, valores y voluntad en la naturaleza. La naturaleza sólo puede ser prescriptiva, si presuponemos que en ella hay una voluntad que establece fines y pondera acciones. En otras palabras, toda posición iusnaturalista es una forma de animismo, es una interpretación social de la naturaleza y, en última instancia, peca de presupuestos teológicos ${ }^{17}$.

Al considerar un sistema normativo natural debemos admitir un sistema de valores universales. Si admitimos un sistema de normas de origen natural, deberíamos

fundamental es una hipótesis que no brinda conocimiento teórico -conocimiento efectivo sobre el derecho- pero es condición de posibilidad del conocimiento del sistema normativo positivo.

Ahora bien, la gran diferencia que habría entre Kant y Kelsen es que para éste último el conocimiento que se posibilitaría de esta forma en el terreno del derecho sí sería conocimiento teórico legítimo. En cambio, el conocimiento posibilitado por la idea trascendental de la libertad en el terreno de la filosofía kantiana es conocimiento práctico, nunca conocimiento teórico en sentido estricto, conocimiento vinculado con lo que la razón debe creer y lo que el ser humano debe hacer, no con una descripción de lo que ontológicamente es.

Creo que esto pone a la norma fundamental como hipótesis o presupuesto trascendental más cerca del neokantismo que de la filosofía kantiana misma. En este sentido, creemos que sería posible pensarla como una condición trascendental y epistemológica que posibilita la existencia del conocimiento científico en el terreno de lo jurídico. Como si dijéramos, es condición de posibilidad del conocimiento del sistema normativo la suposición de la validez del sistema normativo en cuestión. Esto no sería una suposición de un científico en particular o de un sujeto cognoscente en particular. Se trataría de una condición de posibilidad necesaria de todo el terreno epistemológico en el que se da el conocimiento teórico jurídico. Por eso, se la cataloga de trascendental: se trata de un elemento necesario que es condición de posibilidad del conocimiento del objeto fundado por ella y que se encuentra en un nivel diferente de él. En este sentido, no es una mera hipótesis planteada de manera contingente, sino que es una asunción necesaria que hace posible todo conocimiento de lo normativo.

${ }^{15}$ Kelsen, Hans, ob cit., pp. 41-42, 115.

${ }^{16}$ Kelsen, Hans, "La doctrina del derecho natural ante el tribunal de la ciencia", Qué es Justicia, Ariel, Barcelona, 1982, pp. 64-65, 69.

${ }^{17}$ Ibid., p. 65. Kelsen, Hans, Teoría pura del derecho. Op. cit., pp. 23-24. 
admitir también una serie de valores de carácter universal, es decir, válidos de manera perpetua y para todo ser humano. En oposición a este sistema normativo natural, el derecho positivo se presenta como un sistema normativo creado por convención y voluntad humana, que establece una serie de valores cuya validez es relativa al sistema normativo que los postula. Es decir, los valores protegidos y postulados por el sistema normativo positivo habrán de valer para la comunidad sujeta a dicho ordenamiento jurídico y con los límites que posea la validez del sistema normativo en cuestión. Al rechazar la existencia de un ordenamiento normativo natural, Kelsen sólo admite la existencia de sistemas normativos positivos que presentan un conjunto de valores relativos a cada sistema. Esto impide realizar juicios de valor que no sean relativos a un sistema normativo. Resulta imposible juzgar la justicia o injusticia de un sistema normativo, en tanto todos los valores axiológicos por medio de los cuales ponderamos a un sistema son válidos en relación con un determinado sistema normativo presupuesto.

La Teoría pura del derecho de Kelsen no ofrece especificaciones en lo que se refiere al origen de los valores comunitarios.La Teoría pura no se propone ofrecer una explicación histórica, antropológica o epistemológica sobre el origen de los valores. Para las intenciones de Kelsen, basta con establecer una clara delimitación y diferenciación entre derecho y moral, aunque Kelsen no niega que las normas y valores morales pueden ser recibidas en el derecho positivo ${ }^{18}$.Pero habida cuenta de la separación entre derecho y moral, cabe indicar que desde la perspectiva metodológica adoptada por la Teoría pura, tendremos un sistema de valores jurídicamente pertinentes cuando se cuente con un sistema normativo jurídico (es decir, coactivo), con independencia de la existencia de un sistema de valores morales. Insisto en señalar que esto no es una consideración histórica o antropológica. Es decir, no se trata de evaluar desde cuándo hay valores para una comunidad, sino, en todo caso, desde cuándo hay valores jurídicos para una comunidad, desde cuándo hay valores plasmados en un sistema normativo coactivo. En tanto se trata de una teoría del derecho, nos importa únicamente considerar los valores en tanto valores de un

\footnotetext{
${ }^{18}$ Más abajo abordaremos lo que Kelsen llama "delegación entre derecho y moral", Ibid., p. 45.
} 
sistema jurídico. Desde este punto de vista, un valor es tal cuando una norma jurídica así lo reconoce.

La adopción de semejante perspectiva supone desembarazarse de todo presupuesto teológico y permite explicar por qué distintas comunidades tienen diferentes ponderaciones sobre las mismas acciones. En tanto el sistema de valores es dependiente del sistema normativo jurídico positivo, creado por la voluntad humana, no es necesario postular una fuente trascendente para tales valores ni atribuirles un carácter universal. El sistema de valores es convencional, dependiente de la voluntad comunitaria. Por el contrario, si suponemos que los valores se originan en un sistema normativo natural, tendremos la ventaja de postular una serie de elementos axiológicos y prescriptivos exteriores a la norma jurídica positiva que establecería un parámetro para juzgar axiológicamente al derecho positivo. De igual forma, este supuestopermitiría decir que el derecho positivo no crea valores, sino que reconoce valores cuya vigencia es anterior.

Lo cierto es que esta contraposición dicotómica planteada por Kelsen pasa por alto una posición intermedia que tiene aptitudes explicativas en lo que se refiere a la validez de los valores plasmados en el sistema normativo positivo, como así en lo referente a la posibilidad de juzgar la legitimidad y justicia del sistema normativo en cuestión, sin retrotraerse a una posición iusnaturalista. Esta posición superadora de la dicotomía trazada por Kelsen se encuentra en la obra de Max Ernst Mayer.

\section{III- El valor y la norma según Max Ernst Mayer}

En Normas jurídicas y normas de cultura ${ }^{19}$, Max Ernst Mayer propone una forma de concebir la relación entre norma y valor que se aleja de la propuesta por Kelsen. Se trata de una teoría que pone en diálogo a las normas jurídicas con normas culturales extrajurídicas, lo que permite analizar la interacción entre el derecho y otros sistemas normativos, con la particularidad de que los sistemas normativos extrajurídicos juegan un papel fundamental a la hora de fundar la validez del orden jurídico. Por otra parte, la relación entre normas culturales y normas jurídicas concebida por Mayer brinda una nueva explicación de la relación entre el derecho y el

\footnotetext{
${ }^{19}$ Mayer, Max Ernst, Normas jurídicas y normas de cultura, Hammurabi, Buenos Aires, 2000.
} 
valor. En la Teoría pura de Kelsen, no encontramos una preocupación por establecer el origen de los valores comunitarios plasmados en el derecho. Como hemos podido ver, Kelsen no tiene un especial interés en precisar las relaciones entre derecho y moral (u otros sistemas normativos extra-jurídicos), motivo por el cual los valores postulados por el ordenamiento jurídico deben ser tenidos como estrictamente jurídicos y considerados con independencia de su pertenencia a la moral. Es decir, los valores postulados por el orden jurídico valen porque $-\mathrm{y}$ en tanto- pertenecen al orden jurídico, más allá de su presencia o ausencia en sistemas normativos como la moral o la religión. En consecuencia, desde la perspectiva propuesta por Kelsen, no es relevante analizar si dichos valores también se encuentran plasmados en tales sistemas normativos. Se trata de valores que valen -apoyados en la amenaza de la coacción- en tanto se encuentran presentes en normas del orden jurídico. La fuente de los valores es, en consecuencia, el derecho.

La teoría de Mayer presentará una novedad frente a la Teoría pura de Kelsen. En el esquema de Mayer se considera a la norma jurídica en relación con normas culturales no jurídicas. Estas normas no jurídicas son fundamentales para establecer la validez de la norma jurídica. En consecuencia, el valor no jurídico es relevante para determinar los valores jurídicos.

Max Mayer comienza por diferenciar la norma jurídica de la norma cultural ${ }^{\mathbf{2 0}}$. En una comunidad determinada no se encuentra sólo la norma jurídica, sino también

\footnotetext{
${ }^{20}$ Ibid., pp. 55-56. Kelsen admitiría perfectamente el supuesto de distinguir entre diferentes tipos de norma positiva. En una comunidad, pueden encontrarse vigentes varios sistemas normativos positivos que operan en paralelo, como por ejemplo, la moral, la religión y el derecho. Aunque cuenten con elementos distintivos que los diferencian, cada uno de ellos es un sistema de normas que puede coexistir con los restantes.

Ahora bien, como habíamos adelantado, Kelsen ignora deliberadamente la dinámica o interacción que pueda darse entre los distintos sistemas normativos. Kelsen no se interesa por explicar cuál es la dinámica por medio de la cual el derecho, la moral, la religión y cualquier otro sistema normativo no jurídico interactúan. Sin embargo, sí explica un supuesto de tal interacción: cuando el derecho positivo explícitamente dispone que la moral -o cualquier otro sistema normativo positivo no jurídico- sea fuente de derecho. En este sentido, es especialmente ilustrativa la imagen que nos propone Kelsen: "como todo lo que tocaba el rey Midas se transformaba en oro, todo objeto al cual se aplica el derecho adquiere una existencia jurídica" Kelsen, Hans, Teoría pura del derecho, op. cit. p. 126. Es decir, todo aquello a lo que refiera el derecho y que el derecho señale como fuente jurídica, se convierte ipso facto en derecho. A esto llama Kelsen relación de delegación entre derecho y moral, Ibid., p. 45.

Un segundo supuesto de interacción entre derecho y moral es explicado por Kelsen, pero con fines por completos opuestos a los que aquí nos interesan: Kelsen señala que desde la moral se puede emitir un juicio de valor sobre el derecho vigente, pero esto supone y exige que se pueda diferenciar la norma moral de la norma jurídica, para lo cual debe suponerse también que dichas normas establecen
} 
se encuentran otras normas a las que llamaremos normas culturales. Las normas culturales son anteriores a la norma jurídica y son, en principio, independientes de estas últimas. Las normas culturales son aquellas que establecen cuáles son las conductas prescriptas para cada tipo de individuo (según su condición, oficio, pertenencia y demás características) que forma parte de la comunidad. La norma cultural está dirigida a los miembros de la comunidad e indica qué deben hacer, qué se espera de ellos, y supone una serie de valores que se encuentran vigentes. Se incluye entre las normas culturales, las normas morales, religiosas, las prácticas habituales de un oficio, etc ${ }^{21}$.

En oposición a la norma cultural, contamos con la norma jurídica. La norma jurídica es el producto de un lento proceso histórico de codificación de normas culturales. A lo largo de la historia y del desarrollo de las comunidades, la norma jurídica se autonomiza de las restantes normas culturales -a las que originalmente se encontraba unida e incluso identificada - a través de su escritura y volcándose en una nueva forma y con garantías particulares. Se caracteriza por la imposición de sanción y por el establecimiento de garantías precisas como condición de su aplicación ${ }^{22}$.

Una particularidad clave en la diferenciación entre norma jurídica y norma cultural es que aquella no se encuentra dirigida a la comunidad. No es una prescripción destinada al público en general. Es una prescripción que compete únicamente a los funcionarios del Estado. Es una orden dirigida a los jueces y demás órganos encargados de aplicar el derecho. Más aun, Max Mayer cree que el famoso supuesto jurídico según el cual el derecho se reputa conocido por todos es una mera ficción insostenible

mandatos diferentes, es decir, poseen contenidos diferentes, cfr. Ibid., pp. 45-46. Supuesto que a nuestro criterio puede ser cuestionable: no es necesario suponer que la norma jurídica y la norma moral imponen deberes diferentes, sino que basta con admitir que poseen formas diferentes (la norma jurídica puede ser reformulada respetando el principio de imputación y asociándose así un acto coactivo al acto prohibido, mientras que la norma moral sólo prohíbe un acto sin imputar sanción). En este sentido, puede tomarse el ejemplo de Kant, en la Metafísica de las costumbres, donde afirma que los deberes impuestos por el derecho y la moral no son distintos, sino que son tratados por legislaciones distintas (Kant, Immanuel, Metafísica de las costumbres, Altaya, Barcelona, 1996, p. 25). En el caso de Kelsen, se podría admitir que norma jurídica y norma moral poseen un contenido diferente, si como contenido de la norma se considera, precisamente, a la imposición del acto coercitivo característico de la norma jurídica.

${ }^{21}$ Mayer, Max Ernst, Normas jurídicas y normas de cultura, op. cit. pp. 58, 59-60, 136, 144-145.

${ }^{22}$ Ibid., pp. 58-59. 
fácticamente ${ }^{23}$. Sin embargo, tampoco considera Mayer que el derecho necesite ser conocido por parte de la población general para que ésta ajuste su conducta a derecho.

La posición sostenida por Max Mayer genera al menos dos inquietudes. En primer lugar, cómo pueden las prescripciones del derecho tener legitimidad para el pueblo si él no las conoce. Como resulta evidente, una orden obliga a su destinatario siempre que el destinatario sea consciente de la orden recibida. Sólo se puede esperar razonablemente que el destinatario acate la orden y se ajuste a lo estipulado por ella, si, en efecto, recibe la orden. Junto a este problema, se presenta una segunda cuestión: el pueblo no sólo no conoce el derecho, sino que ni siquiera es el destinatario de la prescripción jurídica. Entonces, ¿por qué puede aplicársele legítimamente los castigos impuestos por el derecho, si el derecho consta de órdenes que no están dirigidas a la población en general? Ambos problemas apuntan a una misma cuestión: por qué el derecho sería legítimamente aplicado sobre una población que no lo conoce y a la que él no está dirigido.

Max Mayer ofrece una respuesta recurriendo a las normas culturales. Las normas jurídicas son normas dirigidas a los funcionarios estatales, pero que responden a lo estipulado por las normas culturales. Es decir, las normas jurídicas plasman a través de una técnica legislativa precisa y con carácter coercitivo las prescripciones ya vigentes en las normas culturales. De esta manera, el ciudadano común no necesita poseer un conocimiento técnico específico para saber cuáles son sus deberes, así como tampoco puede excusarse del incumplimiento de sus deberes invocando no conocer el derecho, pues las obligaciones y deberes que posee según el derecho son las mismas que posee de acuerdo con las normas culturales.

La relación que Max Mayer establece entre normas jurídicas y normas culturales permite resolver algunas de las inquietudes que surgen al considerar la distinción trazada por Kelsen entre un sistema de valores absolutos y un sistema de valores relativos.

En primer lugar, la relación entre norma cultural y norma jurídica permite establecer una serie de valores extrajurídicos que permite ponderar y juzgar al sistema

\footnotetext{
${ }^{23}$ Ibid., pp. 45, 49-50, 69-70, 80.
} 
normativo jurídico. A diferencia de lo que ocurre en la teoría de Kelsen, no es necesario partir por considerar a los valores como intrínsecamente jurídicos. Los valores son comunitarios, pero pre-jurídicos, lo que nos permite evaluar axiológicamente al derecho a través de un sistema de valores legítimo para la comunidad, pero no dependiente del derecho mismo. Ahora bien, cabe aclarar que Kelsen acepta un sistema de valores extrajurídicos, plasmado en el ordenamiento moral. Sin embargo, los juicios de valor basados en normas morales no tienen validez jurídica ni ningún tipo de relevancia epistemológica en lo que se refiere a nuestro conocimiento del derecho ${ }^{24}$.

El esquema planteado por Mayer es, en este sentido, distinto. El juicio de valor que se lleva a cabo basándose en las normas culturales debe concordar con las ponderaciones jurídicas de la acción. Como la norma jurídica es válida en tanto concuerda con normas culturales precedentes, la evaluación axiológica llevada a cabo por el derecho debe concordar con la valoración axiológica que postulan las normas culturales por las que se rige el ciudadano común. De esto debe deducirse que el sistema de valores extra-jurídicos - plasmado en las normas culturales- tiene carácter prescriptivo para el ordenamiento jurídico.

En segundo lugar, la separación entre norma cultural y norma jurídica permite plantear el problema de la validez desde un nuevo punto de vista. Tal como ocurre en la Teoría pura del derecho, la validez de la norma jurídica depende de un elemento normativo ulterior. Pero dicho elemento normativo no será jurídico, sino cultural. El problema que se plantea en el esquema kelseniano es que la norma jurídica debe recibir la validez de un elemento normativo jurídico ulterior, lo que conduce a un proceso ascendente de sucesivas transferencias de validez que concluye en la

\footnotetext{
${ }^{24}$ Un juicio basado en el sistema moral positivo $X$ que versa sobre el sistema normativo jurídico $Q$ sólo describe si una norma del segundo está en concordancia con una norma positiva del primero. Se trata, según Kelsen, de juicios de hecho. Pero, vale aclarar, se trata de juicios de hecho que no brindan ningún conocimiento sobre el derecho ni tienen ninguna función o legitimidad al interior del derecho -a menos que el derecho explícitamente remita a la moral como fuente de derecho-. Son enunciados sin ninguna función normativa al interior del derecho y sin ningún valor epistemológico en relación con el derecho. Frente a estos, Kelsen distingue los que considera verdaderos juicios de valor. Los juicios de valor se encuentran basados en normas supuestas. Es decir, cuando un individuo supone que hay una norma vigente que impone una prescripción y juzga una conducta o una norma en relación con dicha norma supuesta. En este caso, se trata de un juicio de valor, puramente subjetivo y fundado en motivaciones psicológicas. Se trata de un juicio de valor porque no describe la concordancia de una acción o norma con una norma positiva, sino que se trata de una ponderación sobre qué norma debería estar vigente, qué norma debería existir. Cfr. Kelsen, Hans, Teoría pura del derecho, op. cit., pp. 47-48.
} 
necesidad de hacer intervenir a la eficacia del sistema normativo, elemento que ya no es en sí mismo normativo, sino meramente fáctico. En cambio, en el esquema propuesto por Max Mayer, la norma jurídica recibiría su validez no de una cuestión de hecho (el acatamiento o aplicación de la norma), sino de otra norma proveniente de un sistema normativo precedente al derecho. De esta manera, Mayer evita el pasaje cuestionable del ser al deber ser, el pasaje de la eficacia a la validez y se mantiene en el terreno de las prescripciones. Sobre este punto volveremos de inmediato.

El esquema planteado por Kelsen establece una dicotomía infranqueable entre derecho positivo y derecho natural, acompañada de una segunda dicotomía: un sistema de valores relativo opuesto a un sistema de valores absolutos. El esquema de Mayer ofrece una posición intermedia con sus propias ventajas. La distinción entre normas culturales y normas jurídicas implica que las normas culturales establecen el parámetro axiológico desde el cual evaluar la justicia y legitimidad del derecho positivo. En este sentido, puede decirse que el conjunto de normas culturales establece un sistema de valores que, por un lado, resultan relativos (originados en la voluntad de la comunidad, de manera convencional y válidos exclusivamente para ésta), pero que por el otro son absolutos en relación con el derecho. Al no originarse en el derecho, tales valores no pueden ser alterados o derogados por él. Debe reconocerlos y sólo será legítimo en cuanto concuerde con las normas culturales. De esta manera, el esquema propuesto por Mayer supera en ciertos aspectos al esquema kelseniano: Por un lado, hay un sistema de valores preexistente al derecho que permite una ponderación axiológica jurídicamente vinculante -epistemológica y prescriptivamente hablando- del derecho; por el otro, no hay necesidad de asumir que los valores son universales o naturales, sino que basta con que sean relativos a dicha comunidad y absolutos para el derecho.

No obstante, el esquema de Mayer queda expuesto a dos críticas. En primer lugar, la teoría propuesta por Mayer deja abierta la puerta a la pregunta por la validez de la norma cultural. Si la validez de la norma jurídica descansa en el sustento que le provee la norma cultural, cabe preguntar por el fundamento de validez de la norma cultural misma. A pesar de tratarse de un filósofo neokantiano, Mayer no ofrece un tratamiento teórico de las condiciones de posibilidad de la fundamentación del ámbito 
de la normatividad. Por el contrario, el tratamiento que hace de las normas culturales parece ofrecer una fundamentación historiográfica, sociológica o antropológica: la validez de la norma cultural depende de una vida común, de la participación en una comunidad cultural $^{25}$. Es decir, la legitimidad de la norma cultural se basa, en última instancia, en una praxis y no en una norma que regula la atribución de validez.

En segundo lugar, la teoría de Mayer puede ser objeto de una crítica interna. Mayer admite que la norma jurídica puede, en ocasiones, ser dictada para imponer un cambio en la conducta de la población. El derecho puede ser un instrumento de cambio cultural. La norma jurídica puede imponer conductas que no son debidas culturalmente, pero que pasarán con el tiempo a ser parte de las normas culturales admitidas en razón del derecho vigente ${ }^{26}$.

Ahora bien, si se admite esta alteración de la relación normal entre norma jurídica y norma cultural, se vuelve necesario volver a preguntar por la fuente de legitimidad de la norma jurídica en cuestión, es decir, de la norma jurídica que tiene como pretensión fundar una nueva pauta cultural hasta el momento inexistente. ¿’Por qué serían válidas las normas jurídicas que intentan provocar cambios culturales en la comunidad, si no están destinadas a la población, no son conocidas por ella y no se sustentan en normas culturales precedentes? Si se tratara de meramente describir hechos, debe decirse que Mayer está en lo cierto:en efecto, en ocasiones las normas jurídicas son capaces de provocar cambios en los hábitos, valores y conductas de la comunidad y, en efecto, en ocasiones las normas jurídicas se distancian de lo impuesto por las pautas culturales admitidas en la comunidad. Pero aquí no se trata de describir prácticas legislativas, sino de evaluar los parámetros de legitimidad de una norma jurídica vigente.

Es en este sentido que la propuesta de Mayer se revela insuficiente. Si bien el autor habla de una aclimatación de las normas jurídicas a la cultura, aclimatación por medio de la cual las normas jurídicas extrañas a la cultura lograrían ganar su lugar al interior de la vida comunitaria, lo cierto es que Mayer no explica cuál es este mecanismo que haría que estas normas sean válidas. Creo que para poder dar una respuesta a esta pregunta debe tomarse en consideración un enfoque dialéctico sobre

\footnotetext{
${ }^{25}$ Cfr. Mayer, Max Ernst, Normas jurídicas y normas de cultura, op. cit. pp. 57-58.

${ }^{26}$ Ibid., pp. 61-62.
} 
el fenómeno jurídico. Sostendremos que hay aspectos de la dialéctica hegeliana particularmente de la Filosofía del derecho y de la Fenomenología del espíritu - que podrían completar y esclarecer el esquema propuesto por Mayer. Más aun, considero que en ciertos respectos, la propuesta de Mayer no puede sino aceptar cierta caracterización típicamente hegeliana de la comunidad en lo que respecta a su aptitud constituyente de normatividad.

\section{IV- Integración de una mirada dialéctica al esquema de Max Mayer}

Quisiera comenzar esta última sección presentando los dos elementos fundamentales que a mi criterio hacen necesario introducir una consideración dialéctica sobre la obra de Max Ernst Mayer aquí considerada, a pesar de que no encontramos en ella referencias al pensamiento de Hegel.

Creo que la obra de Mayer aquí analizada se encuentra con dos problemas que no pueden ser resueltos sin introducir una caracterización dialéctica de la sociedad y de su aptitud para constituir normas. En primer lugar, se trata de la inversión de la relación normal de fundamentación entre norma cultural y norma jurídica. Como ya hemos explicado suficientemente en el apartado anterior, Mayer admite la legitimidad de un fenómeno jurídico peculiar: cuando la norma jurídica no se sustenta en la norma cultural, sino que se dicta con autonomía de ella y pretende generar cambios en el terreno cultural. Este fenómeno jurídico no parece especialmente preocupante para Mayer. Pero a nuestro criterio, genera una paradoja compleja: implicaría que o bien la norma jurídica posee una fuente de validez y legitimidad autónoma a la norma cultural (lo que volvería superflua la teoría de Mayer) o bien se trataría de un fenómeno jurídico que Mayer toma como legítimo, pero que es incapaz de justificar teóricamente.

El segundo problema es que el esquema de Mayer parece suponer $-\mathrm{y}$ requeririnstancias mediadoras entre el individuo y la comunidad como un todo, pero Mayer no explicita la existencia, la función ni la legitimidad de estas instancias mediadoras. Estas instancias mediadoras que Mayer omite analizar tienen -respetando el propio esquema de Mayer- un rol fundamental en la constitución de la normatividad en la esfera cultural, pues Mayer parece referir sutilmente a ellas cuando se trata de 
establecer las normas culturales que rigen los oficios, las profesiones, los ritos religiosos, etc. ${ }^{27}$ Pero su función no concluye aquí. De admitirse tales instancias mediadoras entre los individuos y la comunidad como un todo, capaces de constituir normatividad, se podría admitir una dinámica de diferenciación interna en la comunidad que explicaría el origen de las normas jurídicas como un caso -no tan distinto- de la normativa cultural. De esta forma, se encontraría una nueva explicación a la inversión de la relación normal entre norma cultural y norma jurídica, pues el Estado -con la norma estatal, i.e. jurídica- pasaría a ser una faceta particular (entre otras) de la aptitud constitutiva de normatividad de la comunidad. En esto jugaría un papel fundamental la noción de dialéctica como una nota constitutiva de la comunidad y del modo de despliegue de su aptitud de conformar normatividad.

La noción de dialéctica es una noción sumamente compleja. Aquí no podemos ofrecer una historia del concepto. Basta para nuestros fines entender a la dialéctica como una nota distintiva de la racionalidad, que se caracteriza por constituir a su objeto de conocimiento bajo la forma de un sistema en el cual cada elemento se encuentra relacionado con y determinado por los restantes elementos, sistema que admite un proceso dinámico de crecimiento y formación, y en el que la negación u oposición se presenta como una instancia de su desarrollo. Sostenemos que una caracterización dialéctica de la comunidad en su actividad constitutiva de normatividad será pertinente para esclarecer el esquema de Mayer y resolver los dos problemas antes planteados.

Comencemos por el primer problema. Una teoría que pretenda responder a la pregunta por la validez de las normas en el terreno de lo estrictamente normativo sólo parece poder encontrar la respuesta buscada recorriendo las relaciones entre normas, sin acudir a cuestiones de facto. El esquema de Mayer parece partir del presupuesto según el cual la dicotomía entre normas culturales y normas jurídicas viene acompañada de una dicotomía entre comunidad (pueblo o sociedad, aquí los entendemos como sinónimos) y Estado. Mientras las normas culturales parecen estar íntimamente vinculadas a la historia, identidad y modo de vida de la comunidad, las normas jurídicas provenientes del Estado parecen ser creaciones artificiales, menos

\footnotetext{
${ }^{27}$ Ibid., pp. 58, 61 .
} 
vinculadas a la vida comunitaria que a la administración del poder estatal y su violencia. Esto, por cierto, habría que afirmarlo de manera relativa, pues Mayer señala que el Estado y la norma jurídica se encuentran relacionadas con la vida cultural de la comunidad, aunque con posterioridad se autonomizan de ella. A pesar de esto, la norma emanada del Estado -la norma jurídica- parece tener un carácter secundario y artificial. Si bien la norma estatal concuerda con el modo de vida de la comunidad, lo hace porque sólo repite la norma cultural. Es decir, la norma jurídica o estatal no ofrece un contenido nuevo, sino que vuelve la materia de las normas culturales en una nueva forma. En segundo lugar, la norma jurídica está destinada al funcionario, no a la comunidad. A lo que, en tercer lugar, podría agregarse: la norma tampoco emana de la comunidad, sino del Estado. Por último, como ya hemos dicho, su validez está sujeta a condición de adecuarse a lo pre-estipulado por las normas culturales. Todo esto sugiere que el Estado y la norma jurídica son una instancia de normatividad de categoría inferior y de carácter artificial y limitado.

En oposición a la norma jurídica, la norma cultural encuentra su legitimidad en ser expresión de una vida comunitaria que Mayer parece pensar de manera unitaria. Mayer utiliza la palabra "pueblo", lo que da la idea de una unidad natural ${ }^{\mathbf{2 8}}$. Es decir, una comunidad conformada por un grupo homogéneo, con una historia común, un origen común, una religión común, etc. Ahora bien, aun una vida comunitaria -ajena al fenómeno multicultural actual- requeriría de una subdivisión interna, de una especificación y fragmentación en corporaciones diversas para alcanzar los fines perseguidos por la comunidad concebida como un todo.

Mayer se refiere de forma indeterminada a las distintas instancias de normatividad que componen la comunidad (se habla de "sociedades" en las que se fragmenta la "sociedad" 29 ), aunque en el texto se encuentran algunas referencias explícitas a la corporación de artesanos, marinos, comerciantes, policía, ejército, clero, profesionales de la salud, entre otras ${ }^{30}$. Cada una de estas corporaciones tendría la

\footnotetext{
${ }^{28}$ En Ibid., p.58, Mayer habla del pueblo o clan para referir a la unidad originaria de la comunidad que luego se dividirá en pluralidad de sociedades (corporaciones). Sin embargo, aunque también habla de comunidad o comunidad cultural, vuelve a lo largo del texto a utilizar el término "pueblo", vocablo que parece haber sido usado para referir a una unidad natural.

${ }^{29}$ ibid., p. 58.

${ }^{30}$ Ibid., pp. 61, 142.
} 
aptitud de conformar el conjunto de normas culturales destinadas a quienes cumplen la función pertinente al rubro referido.

Todo esto hace ver que las diversas normas culturales a las que refiere Mayer tienen distintas fuentes, de las que ninguna o casi ninguna es la comunidad en su conjunto. Es decir, si bien en un principio se nos presenta al "pueblo" como una entidad unitaria (como una sustancia ética, si utilizáramos terminología hegeliana), cuando miramos las normas culturales y sus fuentes en detalle apreciamos que no es la comunidad como un todo su fuente, sino que entre la comunidad y el individuo concreto que respeta las normas culturales (y en el que las normas culturales viven) debe intermediar la figura de la corporación y que son las diferentes corporaciones la fuente real de la plural y diversa normatividad cultural.

Si la comunidad es un sujeto legítimamente capaz de constituir normatividad comunitaria en función de su modo de vida, lo cierto es que este modo de vida se presenta expresado en facetas, fragmentado en diversos grupos que requieren plasmar su propia normatividad en pos de cumplir con su función particular y con ella hacer su aporte al todo comunitario ${ }^{31}$. A lo que se suma que difícilmente sea la comunidad como un todo la fuente de normas culturales tan concretas y específicas como aquellas que se refieran a las prácticas comerciales, a los protocolos médicos y científicos, a las reglamentaciones académicas, etc. Todo esto significa que las corporaciones se encuentran legítimamente facultadas a constituir normatividad comunitaria, al menos tanto como se acepte que la comunidad misma lo está.

Si admitimos una instancia mediadora entre la comunidad y el individuo y admitimos a esta instancia mediadora como fuente de normatividad cultural, la dicotomía tajante que Mayer traza entre pueblo y Estado puede debilitarse. Podemos pensar al Estado no como una conformación artificial, opuesta y diferenciada del pueblo, destinada a una tarea ajena a los intereses comunitarios y separada del modo de vida de la comunidad, sino que la podemos pensar como una instancia normativizadora más, una entre tantas otras. Así como la sociedad se fragmenta a sí misma -en razón de la propia necesidad de alcanzar sus metas colectivas- en una pluralidad de corporaciones e instituciones normativizadoras, el Estado puede ser

\footnotetext{
${ }^{31}$ Hegel, G. W. F., Principios de la filosofía del derecho, Buenos Aires, Sudamericana, 2004, pp. 222-223, 225. §§250, 252, 256.
} 
gestado como una necesidad más del modo de vida de la comunidad y como una instancia mediadora más. Cabe aclarar que para Hegel el Estado no sería una corporación, sino una expresión del todo comunitario que supera la individualidad del sujeto singular y la particularidad (todavía no completamente universal) de la corporación $^{32}$. Para nuestro análisis, no es necesario emprender una discusión sobre la naturaleza precisa del Estado. Basta con explicar la posible articulación y relación entre normas culturales y normas jurídicas desde una perspectiva dialéctica.

Si aceptamos pensar al Estado como una faceta más de la comunidad en su auto-división en corporaciones e instituciones capaces de conformar normatividad comunitaria, cabe admitir a las normas jurídicas como un caso más de normas culturales. Es decir, nuevamente, la dicotomía tajante que Mayer presenta entre norma cultural y norma jurídica se cae o se debilita. La separación que habría entre una norma cultural y una norma jurídica no sería de otra naturaleza o cualitativamente diferente a la que habría entre dos normas admitidas como culturales: por ejemplo, la distinción entre una norma penal y una norma religiosa no es mayor a la distinción que hay entre esta norma religiosa y una norma que rija el ejercicio del comercio o el correcto desempeño en el ámbito académico y científico. Obviamente, se puede admitir que el Estado tendrá sus particularidades que no serán encontradas en la Iglesia, en la academia, etc. Pero lo cierto es que lo mismo se puede decir de cada una de estas instituciones: Cada instancia mediadora (corporación o institución) tendrá particularidades distintivas que no estarán presentes en las restantes.

Salvada la tajante dicotomía con la que Mayer comienza su análisis, debe cuestionarse un punto central de su propuesta. Si no hay una dicotomía esencial entre la norma jurídica y la norma cultural y aquélla no es más que un caso particular de ésta, entonces carece de sentido explicar la legitimidad de la norma jurídica por medio de una referencia a la norma cultural, a menos que se admita junto con esto que la norma cultural se justifica a sí misma. En otras palabras, si la norma jurídica es una norma cultural, no cabe legitimar la norma jurídica aduciendo que concuerda con las normas culturales. En tanto ella misma es una norma cultural, justificarla apelando a

\footnotetext{
32 Ibid., pp. 227-229. §258.
} 
ella misma exigiría plantear una pregunta por la validez en general de todas las normas culturales. Sin esta respuesta, la justificación de la norma jurídica se vuelve obsoleta.

Ahora bien, ¿̇ónde encuentra Mayer la fundamentación de la legitimidad de la norma cultural? Dicha fundamentación se halla en la vida comunitaria, en el modo de vida del pueblo. Esto supone que el pueblo -a través de sus diversas corporaciones e instituciones- es en esencia capaz de constituir normatividad. De lo contrario, no cabría suponer que una situación de hecho puede convertirse en norma, deber ser. La comunidad, como sujeto portador de una voluntad, es capaz de constituir normatividad para sí misma. Esto se plasmará tanto en las diversas corporaciones que conforman lo que Mayer califica como norma cultural, como también en el Estado. La fundamentación de todas las normas culturales (incluso las jurídicas) puede recaer entonces en un único elemento: en la voluntad comunitaria, que a través de un proceso de diferenciación interna queda expresada en una serie diversa de instituciones, corporaciones y organizaciones. Una de tales instancias de subjetividad será el Estado.

Las normas estatales tendrán, entonces, validez puesto que el Estado es expresión de la voluntad y vida comunitaria. En otras palabras, las normas jurídicas valen porque el Estado mismo se corresponde desde un inicio con el modo de vida de la comunidad, así como el resto de las normas culturales valen porque las instituciones y corporaciones de las que emanan corresponden al modo de vida de la comunidad y a la consecución de sus necesidades. Ahora bien, aunque sería de esperar un cierto grado de concordancia entre las normas culturales emanadas de diversa fuente, lo cierto es que tal concordancia no es estrictamente necesaria. A pesar de que todas las normas culturales emanen, en última instancia, de una fuente común, lo cierto es que podemos encontrar tensión entre normas religiosas, prácticas comerciales, normas jurídicas, etc. Esto quiere decir que la tensión y oposición que podríamos encontrar entre normas jurídicas y normas culturales -tal como lo describe Mayer- también puede encontrarse entre diversas normas culturales. Por ejemplo, la norma religiosa que postula perdonar las deudas se contrapone a las prácticas comerciales que rigen la firma y uso de pagarés, cheques y otros títulos de crédito. 
De esta manera, se ve que la tensión y oposición entre norma jurídica y normas culturales no es propia de una relación particular que habría entre Estado y sociedad. Todas las normas culturales pueden encontrarse en tensión y oposición con otras normas culturales emanadas de diferente fuente. Con lo cual, el estudio de la relación entre la norma jurídica y la norma cultural no es más que un caso entre muchos de cómo normas culturales de distinta fuente pueden interactuar y afectarse. La visión que Mayer tiene de la relación entre norma cultural y norma jurídica, haciendo que la norma jurídica requiera de una tarea de fundamentación especial y poniéndola en una relación de dependencia, se sostiene en una concepción del Estado como una creación artificial que se agrega de manera exterior a la vida de la comunidad. En tanto el Estado es visto como una creación artificial que no emana de la vida misma de la comunidad, de la organización que la comunidad se da a sí misma, la norma jurídica parece necesitar una fundamentación especial -diferente a la fundamentación que se da al resto de las normas culturales-, generándose un problema grave de legitimación para estas normas cuando entran en contradicción con las restantes normas culturales.

Por el contrario, si aceptamos que las normas jurídicas son un caso más de normas culturales, podemos pensar la tensión entre una norma jurídica y una norma cultural como un caso más de contraposición entre normas culturales de distinta fuente. Como hemos indicado más arriba, las normas religiosas pueden confrontar con las prácticas habituales del comercio. Las prácticas hospitalarias y médicas pueden entrar en tensión con normas académicas. La confrontación entre norma estatal (jurídica) y las restantes normas culturales (religiosas, de oficio, de profesión, etc.) no son un caso especial de contraposición entre normas culturales.

En consecuencia, lo que hemos descripto como una relación inversa de influencia entre norma jurídica y norma cultural, en verdad, no es más que un caso en el cual las normas emanadas de una determinada fuente se vuelven capaces de modificar las normas y prácticas ordenadas por otra fuente. Esto supone, precisamente, comenzar a considerar a la totalidad de las normas culturales de una comunidad (provengan de la fuente que provengan) como parte de un mismo sistema que posee un carácter dinámico y dialéctico. Las normas provienen de fuentes diversas, poseen campos de aplicación parcial y finalidades particulares. En su 
articulación conjunta, las normas entrarán en tensión entre sí y pueden provocar variaciones unas en otras: ya sea que algunas normas se vean cuestionadas y erosionadas en su fundamentación y legitimidad, ya sea que otras normas originalmente acotadas a un ámbito específico se extiendan al conjunto total normativo. Podemos suponer que los factores que determinan el modo en que una norma interactúa con las restantes está determinado por su concordancia con la totalidad del sistema, con su habilidad para tender hacia un fin comunitario que supere las metas inmediatas de la institución o corporación de la que ella emana, etc.

Esto significa que una caracterización adecuada de la dinámica de interacción entre las distintas normas culturales no puede pasar por alto el carácter dialéctico de dicha interacción. Esto supone considerar necesariamente al conjunto de normas como un sistema unitario -aunque se componga de normas de diferente fuente-. En segundo lugar, reconocer que el sistema contendrá una dinámica de negación y oposición entre normas. Las normas de diferente fuente pueden contraponerse. Pero, en tercer lugar, el desarrollo del sistema normativo debe tender hacia la conformación de un sistema unitario que supere dichas contradicciones y oposiciones. En esta dinámica, tanto las llamadas por Mayer normas culturales y normas jurídicas se ganarán su lugar en razón de la aptitud que tengan para integrarse en un todo más amplio, en las conformaciones futuras que la totalidad del sistema de normas adoptará. De modo tal que tanto la norma jurídica como la norma cultural pueden quedar sin efecto por su discrepancia con las restantes normas. No sólo la norma jurídica puede ver limitada su autoridad si entra en contradicción con normas culturales generalmente admitidas. Las propias normas culturales podrían sufrir modificaciones si las normas jurídicas novedosas -contrapuestas a aquellas- logran integrarse satisfactoriamente al todo normativo.

De aceptarse el carácter dialéctico de la interacción entre normas de distinta fuente, se aceptaría que la legitimidad de cada uno de los elementos normativos no se encontraría sino en el todo mismo. Es el sistema normativo como un todo compuesto de diversas fuentes normativas, articuladas de diversas maneras, lo que brindaría validez a cada una de las normas. La validez de la norma no radicaría, entonces, en ninguna consideración que pueda hacerse sobre un elemento aislado o en su relación 
con otra norma aislada. La validez de los elementos normativos se establecería en relación con el todo que pueda tolerarlos en una estructura integradora. La validez de la norma estatal puede buscarse en su concordancia con otras normas culturales, así como la validez de cualquier norma cultural puede buscarse en su articulación con las restantes normas culturales -entre las que se cuentan las normas jurídicas (estatales). Esto indica que la pregunta por la legitimidad o validez de las normas jurídicas no conforma una pregunta especial, diferenciada de la pregunta por la validez de cualquier otra norma cultural.

\section{V- Conclusiones}

En la primera sección del presente trabajo consideramos la Teoría pura del derecho de Kelsen y su contraposición entre un sistema normativo positivo y un sistema normativo natural. La oposición entre ellos corría en paralelo a la confrontación entre un sistema de valores convencionales y uno de valores universales, lo que cerraba la puerta a toda otra posibilidad.

Frente a este esquema cerrado, Max Ernst Mayer presenta una vía de escape. Al introducir una consideración sobre la relación de fundamentación entre normas de dos orígenes distintos -normas culturales y normas jurídicas-, Mayer es capaz de presentar al derecho como un sistema de valores relativos, convencionales, pero que se apoya en un segundo sistema de valores comunitarios que lo fundamentan. Este segundo sistema de valores -aunque también producto de la voluntad de la comunidad y, por tanto, en última instancia convencional- se presenta en relación con el derecho con un carácter absoluto, sin implicar esto un retroceso al naturalismo. De esta forma, Mayer presenta un esquema que permite pensar al derecho como un sistema de normas y valores que supera la dicotomía kelseniana y tiene las virtudes propias de ambos sistemas de valores: por un lado, los sistemas de normas son relativos, convencionales, humanos; por el otro, hay frente al derecho un sistema de valores que nos permite juzgarlo, ponderarlo legítimamente, cuestionar su validez, etc.

Ahora bien, el esquema propuesto por Mayer se sostiene en dos tajantes separaciones: una entre Estado y comunidad (o pueblo), otra entre norma cultural y norma jurídica. Mientras la comunidad o el pueblo es la fuente legítima de 
normatividad (normas culturales), el Estado parece ser pensado como una instancia artificial de normatividad; la norma jurídica requiere de una fundamentación de su validez apoyándose en la norma cultural.

Sin embargo, Mayer introduce un fenómeno particular que provoca una rearticulación integral de su esquema. Al suponer una posible relación inversa entre norma jurídica y norma cultural (en la cual la norma jurídica no se apoya en ésta, sino que la contradice e incluso la modifica), se vuelve necesario buscar una fuente de fundamentación de la norma jurídica que no recaiga en las normas culturales. En este sentido, se descubre que Mayer da preeminencia a la validez de las normas culturales frente a las normas jurídicas, en razón de un supuesto no explicitado: la presunta artificialidad del Estado y su carácter exógeno frente al pueblo o comunidad como una unidad natural. La fuente de validez de las normas culturales sólo puede encontrarse en su correspondencia con el modo de vida de la comunidad. Pero la enorme diversidad que se encuentra entre las diferentes normas culturales obliga a admitir la existencia de corporaciones e instituciones mediadoras. Es decir, la comunidad es normativizadora, pero no de forma inmediata ni como un todo homogéneo, sino a través de una pluralidad de instancias particulares y heterogéneas.

Al tener que recurrir - de manera solapada- a la figura de la corporación, esta separación tajante entre comunidad y Estado debe ser reelaborada y cuestionada. Admitido que en el seno de la comunidad surgen múltiples instancias de normativización, el Estado puede ser considerado como una fuente más de normatividad entre otras y la separación tajante entre norma cultural y norma jurídica se cae o se debilita. Si la norma cultural deja de ser considerada como una norma con carácter preferencial (es decir, como la única norma emanada de la voluntad comunitaria como un todo) para pasar a ser la norma expresada por una instancia particular de dicha comunidad, ¿por qué la norma jurídica (estatal) tendría un carácter inferior, menos universal, más artificial frente a las restantes normas culturales emanadas de corporaciones e instituciones? ¿Por qué la norma estatal requeriría de un proceso de fundamentación ulterior, diferenciado del proceso de fundamentación de las normas culturales? 
El reconocimiento de la necesidad de hacer intervenir en el esquema de Mayer a la figura de la corporación y, con ello, integrar a dicho esquema una pluralidad de fuentes normativas, lo que vuelve a la norma estatal una norma cultural más entre varias, conduce a comprender que la relación entre norma cultural y norma jurídica tal como fuera pensada por Mayer- resulta insuficiente. No puede admitirse sin más que ciertas normas culturales tengan preeminencia a la hora de atribuir validez a las restantes normas culturales (i.e. normas jurídicas).Por el contrario, la dinámica a través de la que interactúan de manera multidireccional normas de diversa fuente acarrea que toda norma puede ser derogada o suprimida por un conjunto de otras normas, poniéndose la preeminencia en el todo normativo -conformado por diversas fuentesantes que en un elemento aislado, así como la validez de cada elemento no depende tampoco de su concordancia con un elemento singular destacado ("la norma cultural"), sino con su aptitud para integrarse en el todo. De esta manera, evita darse prioridad a un elemento normativo frente a los restantes y se resuelve la problemática planteada por Mayer: cómo explicar el caso en que la norma jurídica -contraria a la norma cultural- provoca un cambio cultural.

\section{Bibliografía}

Eisler, Rudolf, Lexicon. Online: http://www.textlog.de/32403.html.

Hammer, Stefan, “A Neo-Kantian Theory of Legal Knowledge in Kelsen's Pure Theory of Law?", Paulson, Stanley, Litschewski Paulson, Bonnie, Normativity and Norms. Critical Perspectives on Kelsenian Themes, Oxford, Clarendon Press, 1998, pp. 177-194.

Hegel, G. W. F., Principios de la filosofía del derecho, Buenos Aires, Sudamericana, 2004.

Kant, Imannuel, Crítica de la razón pura, Colihue, Buenos Aires, 2007.

Kant, Immanuel, Metafísica de las costumbres, Altaya, Barcelona, 1996

Kelsen, Hans, Teoría pura del derecho, trad. R. Vernengo, UNAM, México, 1982

Kelsen, Hans, Teoría pura del derecho, Eudeba, Buenos Aires, 2015.

Kelsen, Hans, "La doctrina del derecho natural ante el tribunal de la ciencia", Qué es Justicia, Ariel, Barcelona, 1982, pp. 64-112. 
Luft, Sebastian, The Space of Culture: Towards a Neo-Kantian. Philosophy of Culture (Cohen, Natorp, and Cassirer), Oxford, Oxford Scholarship Online, 2015.

Marí, Enrique, "Racionalidad e imaginario social en el discurso del orden, Doxa, n 3, 1986, pp. 93-111

Mayer, Max Ernst, Normas jurídicas y normas de cultura, Hammurabi, Buenos Aires, 2000.

Paulson, Stanley, "The Neo-Kantian Dimension of Kelsen's Pure Theory of Law", Oxford Journal of Legal Studies, Vol 12, No 3, 1992, pp. 311-332.

Paulson, Stanley, “La alternativa kantiana de Kelsen: Una crítica”, DOXA, n. 9, 1991, pp. 173-187.

Paulson, Stanley, "Faktum/Wert-Distinktion, Zwei-Welten-Lehre und immanenter Sinn. Hans Kelsen als Neukantianer“, Alexy, R., Meyer, L., Paulson, S., Sprenger, G., Neukantianismus und Rechtsphilosophie, Nomos Verlagsgesselschaft, Baden-Baden, 2002, pp. 223-252.

Sprenger, Gerhard, "Die Wertlehre des Badener Neukantianismus und ihre Ausstrahlungen in die Rechtsphilosophie“, Alexy, R., Meyer, L., Paulson, S., Sprenger, G., Neukantianismus und Rechtsphilosophie, Nomos Verlagsgesselschaft, BadenBaden, 2002, pp. 157-178.

Schmill, Ulises, "Algunas influencias de Hermann Cohen en Hans Kelsen", Isonomía, no. 21, México, oct. 2004, pp. 117-155.

Strauss, Leo, "Sobre la ley natural", Persecución y el arte de escribir y otros ensayos de filosofía política, Edicions Alfons El Magnánim, Valencia, 1996, pp. 133-148. 